\title{
Team-Based Learning como Forma de Aprendizagem Colaborativa e Sala de Aula Invertida com Centralidade nos Estudantes no Processo Ensino- Aprendizagem
}

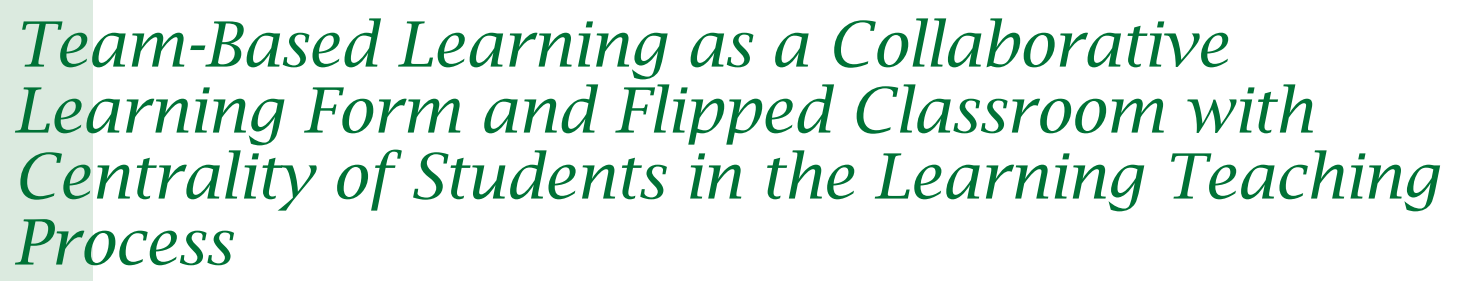

Bruno Luciano Carneiro Alves de OliveiraI Sara Fiterman Lima ${ }^{I}$ Livia dos Santos Rodrigues ${ }^{I}$ Gerson Alves Pereira Júnior II

\section{PALAVRAS-CHAVE \\ - Estudantes de Medicina. \\ - Educação Médica. \\ - Aprendizagem. \\ - Metodologia. \\ - Comunicação em Saúde.}

I Universidade Federal do Maranhão, São Luís, Maranhão, Brasil.

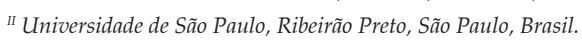




\section{KEY-WORDS}

- Students, Medical.

- Education, Medical.

- Learning.

- Methodology.

- Health Communication.

\begin{abstract}
Teaching methodologies in higher education have evolved in recent years, increasingly emphasizing the learner's role in the teaching-learning process, and the construction of their own knowledge and that of their peers. However, the training structure that exists in the majority of medical education courses still lack innovations in their pedagogical practices that will allow meaningful learning experiences that stimulated dialog-based learning and student interaction. One of the proposals that has been promoted as an alternative for improving medical education is Team Based Learning (TBL), which favors dynamic learning through group discussions, in a motivating, cooperative and supportive environment. In this context, this article aims to describe, through a report of an experiment, the planning, implementation and development of contents on the conception and formation of the human being and reproductive health, using TBL as a teaching methodology on a medical course of the expansion of the Program Mais Médicos [More Doctors Program] in the interior of the Northeast of Brazil. Initially, the students received study scripts on the contents to be developed in the classroom, and performed prior study of the activities to be developed. In the classroom, they took a test, individually. Afterwards, divided into small groups, they debated each of the questions and the answers that they had selected, before coming together to form a consensus, to present a single answer, as a group. TBL was found to be very useful in the learning process, and in the evaluation of the students' training. The students' exercised skills in communication, argumentation and persuasion, improved their interaction between peers and with the faculty members, and enhanced their personal performance. It is believed that TBL offers a more articulated way of gaining the knowledge needed to respond to the health demands and needs of the population, and helping to transform the local conditions of life and health.
\end{abstract}

Recebido em: 20/6/18

Aceito em: 9/7/18

\section{INTRODUÇÃO}

As rápidas e crescentes transformações nas sociedades contemporâneas têm colocado em debate os aspectos relativos à necessidade de mudanças na educação universitária e na formação profissional ${ }^{1,2}$. Na área de saúde, ainda se observa uma formação com predomínio de metodologias tradicionais, influenciadas pelas tendências cartesianas, fragmentadas e reducionistas, em que a dinâmica de ensino e aprendizagem coloca o docente no papel central e de transmissor de conteúdos, e o discente no papel periférico e de mero espectador ${ }^{3}$.

Os cursos de Medicina, por sua vez, caracterizam-se pela interpretação e classificação de fenômenos relacionados à saúde e seleção de intervenções para a resolução dos agravos ou problemas de saúde-doença. Essa condição tem estabelecido a incorporação crescente de tecnologias, levando à formação médica fragmentada em campos altamente especializados, sofisticados e de elevada densidade tecnológica e financeira, que demandam a busca por eficiência técnica ${ }^{4,5,6}$.

Contudo, trata-se de uma estrutura formadora que apresenta fragilidades e contradições importantes, o que revela a necessidade de criar novas formas de trabalhar o conhecimen- to no intuito de alcançar competências e habilidades que recoloquem entre os estudantes a dimensão humana e social no cuidado aos sujeitos que demandam atenção médica ${ }^{4-7}$, sobretudo em contextos socioeconômicos mais desfavorecidos como os observados nas diversas cidades do Estado do Maranhão.

Esse debate tem fomentado a crescente incorporação de metodologias inovadoras de ensino-aprendizagem que admitam uma prática pedagógica ativa, participativa, crítico-reflexiva e edificadora de conhecimentos com base em experiências significativas ${ }^{4,5,6}$. Assim, em todo o mundo ao longo das últimas décadas, na educação das profissões de saúde ocorre um deslocamento dos métodos tradicionais de ensino para as práticas pedagógicas que envolvem ativamente os alunos ${ }^{8,9}$.

Para Moran ${ }^{10}$, a questão posta é que, se desejamos formar estudantes proativos, precisamos adotar metodologias em que estes se envolvam em atividades cada vez mais complexas, onde tenham que tomar decisões e avaliar os resultados com apoio de materiais relevantes.

Na formação médica, essa necessidade de inovação tenta romper paradigmas educacionais arraigados e busca criar importantes movimentos que deslocam o foco do ensinar e 
do professor para a aprendizagem e centralidade no aluno, na perspectiva de desenvolver, nestes, concretos resultados que são observados em habilidades, conhecimentos e atitudes ${ }^{11}$. Assim, a inserção de métodos ativos em cursos de Medicina também se torna um desafio frente à diversidade de novos métodos ativos de ensino disponíveis ${ }^{4}$.

Nesse sentido, as novas Diretrizes Curriculares Nacionais dos Cursos de Graduação em Medicina, publicadas em 2014, trazem em seu escopo que tais cursos devem utilizar metodologias ativas (Artigo 32), privilegiando a participação ativa do estudante na construção do conhecimento e na integração entre os conteúdos (Artigo 29 - II), acolhendo, assim, a relevância de métodos que estimulem a participação efetiva do aluno ${ }^{7}$.

Embora sejam diversas as metodologias e técnicas educacionais construtivistas elaboradas e utilizadas para permitir esta formação, uma das limitações nesse cenário e que merece devido destaque é que a maioria delas demanda turmas ou grupos pequenos e mais professores, sendo um desafio tornar o processo de ensino-aprendizagem dinâmico, significativo e cooperativo frente a turmas grandes ${ }^{12,13}$.
Diante de tal desafio, destaca-se a Aprendizagem Baseada em Equipes (ABE), do inglês Team-Based Learning (TBL), que consiste numa estratégia educacional que propõe aos estudantes uma aprendizagem ativa e que pode ser usada com grandes classes de estudantes divididos em pequenos grupos ${ }^{5,14}$.

Nessa perspectiva, o TBL mostra-se uma boa opção de estratégia educacional para a educação médica, pois permite atuar numa realidade de grandes turmas - 25 a 100 estudantes divididos em grupos de 5 a 7 componentes - e poucos docentes $^{14,15}$. Numa revisão sistemática da literatura de 117 estudos, publicada em 2017, foi observado predomínio da utilização de TBL na educação médica relativamente a outras formações de nível superior em saúde, sendo utilizado para equipes de 3 a 12 estudantes, e em turmas de 101 a 150 alunos $^{8}$.

O TBL foi criado no final dos anos 1970 por Larry Michaelsen com o objetivo de melhorar a aprendizagem e desenvolver habilidades de trabalho colaborativo por meio de estratégias como o gerenciamento de equipes de aprendizagem, tarefas de preparação e aplicação de conceitos, feedback constante e avaliação entre os pares (Quadro 1). O método apresenta quatro

QuAdro 1

Componentes e características gerais do Team-Based Learning (TBL)

\section{Componentes Características gerais}

É uma estratégia de ensino-aprendizagem centrada no estudante. Método educacional para grandes grupos. Coordenado por professor, possibilita a interação e o trabalho em equipe. Ocorre em quatro etapas: 1 - Preparação (pré-classe); 2 - Garantia de

Descrição preparo por meio da aplicação dos testes que asseguram a aprendizagem (em classe) e debate sobre as questões aplicadas em sala; 3 - Aplicação dos conceitos (os 4S: problema significativo, escolha específica, mesmo problema e relatos simultâneos); 4 Autoavaliação e avaliação interpares dos estudantes para observar o cumprimento dos objetivos da discussão do TBL.

Definição de papéis: a instituição deve definir o papel do profissional a ser formado e garantir a infraestrutura necessária

Instituição para sua implementação. Há necessidade de instituir serviços de apoio emocional ao estudante. Promove maior interação entre as diversas disciplinas. Feedback contínuo ao aluno, aos docentes e à instituição, com as correções necessárias. Recupera a dimensão social, crítica e reflexiva necessária à prática médica.

Interação: interage com os estudantes, atuando apenas quando é necessário. Assume papel de facilitador do aprendizado.

Docente Cabe ao professor mediar estímulos e oportunidades para que todos os estudantes possam desenvolver habilidades e atitudes de representatividade, autonomia e comunicação. Em geral, os docentes têm mais trabalho na fase prévia à execução do TBL. Discente crítica e construtiva. Desenvolve a inteligência relacional, autonomia e maior responsabilidade sobre o autoaprendizado. O

Ativo: o foco é desviado para que seja responsável pelo seu próprio ensino. Quando bem orientado, passa a exercer atitude trabalho em grupos ou equipes estimula a organização e o mútuo comprometimento entre os estudantes.

Vantagens

Individualizar as necessidades dos estudantes ao se trabalhar com grupos pequenos, facilitando a interação aluno-professor. O respeito à singularidade e a habilidade de lidar com o outro permitem a aquisição progressiva de autonomia e maturidade.

Desvantagens

Manter treinamento e capacitação docente contínuos. Consome enorme tempo docente de preparo, aplicação e avaliação da atividade. Requer o trabalho com pequenos grupos para que seja efetivo. Requer o sacrifício de se transmitir todo o conteúdo, sendo necessário selecionar o "conteúdo essencial" que será trabalhado exaustivamente.

Inteligência: o trabalho em equipes proporciona o desenvolvimento inter- e intrapessoal, a habilidade de conversar e

Resultados compartilhar que representa a inteligência relacional, que compreende a inteligência intrapessoal (autoconhecimento emocional, controle emocional e automotivação) e a inteligência interpessoal (reconhecimento de emoções de outras pessoas e habilidades em relacionamentos interpessoais).

Para saber mais acesse: https:/ / www.teambasedlearning.org/

Fonte: Adaptado de Bollela et al. ${ }^{5}$; Souza et al. ${ }^{6}$.

REVISTA BRASILEIRA DE EDUCAÇÃO MÉDICA

$\left.88\right|_{42(4): 86-95 ; 2018}$ 
etapas (Figura 1) e proporciona um ambiente motivador e cooperativo, contribuindo para minimizar o desinteresse dos estudantes pelo tema da aula, objetivando que se sintam responsáveis pela própria aprendizagem e pela dos colegas ${ }^{14}$.5,6,16,17,18.

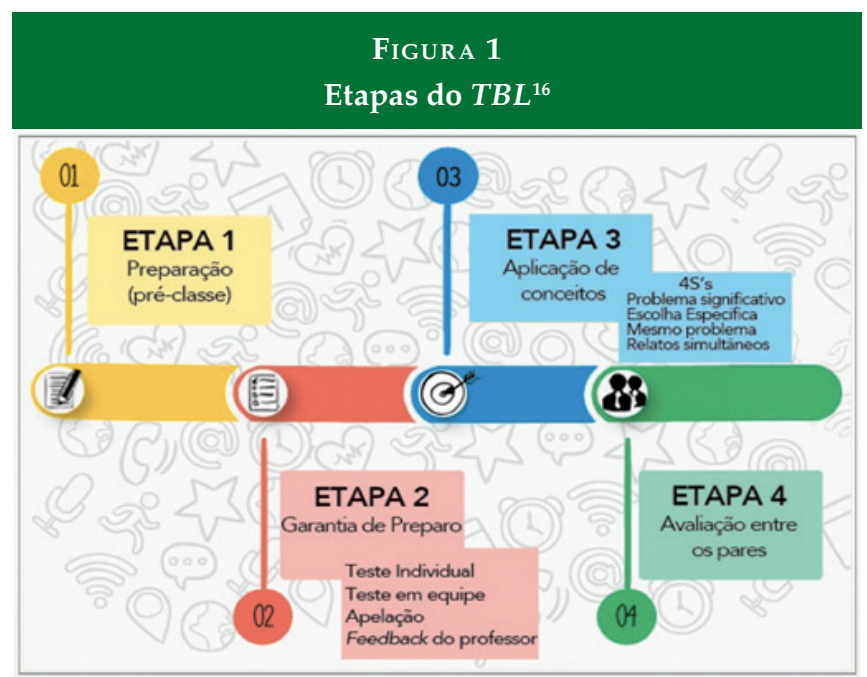

Nesse contexto, este artigo teve como objetivo, por meio de um relato de experiência, descrever o planejamento, a implantação e o desenvolvimento de conteúdos sobre concepção e formação do ser humano e saúde reprodutiva utilizando o TBL como metodologia de ensino em um curso de Medicina localizado no interior do Nordeste brasileiro.

\section{CONTEXTO DA IMPLANTAÇÃO DO TBL}

O campus do curso de Medicina em que foram implementadas as atividades do TBL aqui apresentadas está localizado na cidade de Pinheiro, que fica a 121,6 quilômetros da capital do Estado do Maranhão. Trata-se de um curso de expansão do ensino médico no Brasil iniciado em 2014 e que está vinculado ao Programa Mais Médicos do Ministério da Educação brasileiro (MEC).

Nessa experiência, o TBL foi utilizado para substituir as aulas expositivas, centradas no professor, e para promover aprendizagem ativa e centrada no estudante. Como referencial metodológico para sustentar cientificamente a utilização do $T B L$, consideramos as quatro etapas disponíveis na literatura: (1) preparação individual (pré-classe) como sala de aula invertida; (2) avaliação da garantia de preparo (presencial em sala de aula); (3) aplicação de conceitos (também em sala de aula) e (4) avaliação $0^{5,13,16,17,19}$. Ressalte-se que a etapa 2 deve ter quatro fases: teste individual, teste em equipe, apelação e feedback do professor ${ }^{16}$.
Nessa experiência, estiveram envolvidos quatro docentes que ministraram os conteúdos de Política de Atenção à Saúde do Homem, Política de Atenção à Saúde da Mulher, Rede Cegonha, Infecções Sexualmente Transmissíveis e Direitos Sexuais e Reprodutivos para 38 estudantes do terceiro período do curso, durante o módulo de concepção, formação do ser humano e saúde reprodutiva (Quadro 2).

\section{DESCRIÇÃO DA ESTRATÉGIA TBL}

Foi realizada uma sessão de TBL para cada um dos cinco conteúdos propostos. Em média, cada sessão teve duração de duas horas e meia, e, por se tratar de uma experiência ainda em implantação, os docentes optaram pelo preparo em conjunto dos conteúdos e materiais a serem trabalhados.

Ainda no planejamento entre docentes para organizar a oferta de conteúdos por meio da metodologia escolhida, decidiu-se por consenso pela necessidade de orientar os estudantes sobre a utilização do método, o que inclusive é recomendado na literatura. Dessa forma, inicialmente foram ofertadas aos estudantes informações sobre o TBL, mostrando seus conceitos e características básicas, como suas fases e etapas e o sistema de avaliação, para oferecer uma visão geral dos recursos e benefícios dessa metodologia de ensino para os discentes envolvidos ${ }^{13}$.

Nessa aula inicial, também foi realizada uma importante etapa para a implantação do TBL, que consistiu na formação das equipes. Respeitando os preceitos do método, os estudantes foram divididos em grupos, de forma aleatória e equilibrada (distribuídos pelo sistema acadêmico - Sigaa), buscando evitar o reforço de vínculos afetivos entre os membros (irmãos, namorados, amigos muito próximos), que são reconhecidamente capazes de dificultar o processo, e ainda valorizar e incluir a diversidade na composição dos grupos.

Seguindo para a apresentação da experiência com a aplicação das sessões de $T B L$, descrevem-se na sequência as etapas preconizadas pelo método, entremeando com a descrição das vivências para planejar, implantar e desenvolver os conteúdos de concepção e formação do ser humano e saúde reprodutiva utilizando o TBL.

As sessões tiveram início com a fase de preparação individual. Com uma semana de antecedência foi disponibilizado um roteiro de estudos aos estudantes para leitura prévia, contendo uma revisão teórica dos assuntos, os objetivos de aprendizagem e a indicação de material a ser trabalhado durante a aula. Nessa etapa do TBL, preconiza-se que os estudantes devem ser responsáveis por seu preparo individual por meio de leituras antecipadas ou de outras atividades definidas pelo professor com antecedência. Assim, optou-se sempre pela utilização de materiais escritos, de referência, sobre cada assunto 


\begin{tabular}{|c|c|}
\hline & $\begin{array}{l}\text { QUADro } 2 \\
\text { Conteúdos discutidos no módulo de concepção, formação do ser humano e saúde } \\
\text { reprodutiva por meio do Team-Based Learning (TBL). Curso de Medicina, } 2016\end{array}$ \\
\hline Tema abordado & Conteúdos discutidos \\
\hline $\begin{array}{l}\text { Política de Atenção à } \\
\text { Saúde do Homem }\end{array}$ & $\begin{array}{l}\text { Importância da política de saúde do homem (princípios, diretrizes e estratégias voltadas à melhoria das condições de } \\
\text { saúde dessa população); Perfil de morbimortalidade da população masculina nos cenários nacional, regional e local; } \\
\text { Ações e serviços de saúde e sua organização no atendimento à saúde do homem; Articulação da política de saúde do } \\
\text { homem com outras políticas de saúde, Vulnerabilidade sociais e de saúde da população masculina como critério de } \\
\text { planejamento e programação das ações de saúde dessa população; Monitoramento e avaliação de Indicadores e metas } \\
\text { para a promoção da saúde do homem, e redução das iniquidades regionais e locais. }\end{array}$ \\
\hline $\begin{array}{l}\text { Política de Atenção à } \\
\text { Saúde da Mulher }\end{array}$ & $\begin{array}{l}\text { Importância da política de saúde da mulher (princípios, diretrizes e estratégias voltadas à melhoria das condições de } \\
\text { saúde dessa população); Perfil de morbimortalidade da população feminina nos cenários nacional, regional e local; } \\
\text { Ações e serviços de saúde e sua organização no atendimento à saúde da mulher; Articulação da política de saúde da } \\
\text { mulher com outras políticas de saúde; Vulnerabilidade sociais e de saúde da população feminina como critério de } \\
\text { planejamento e programação das ações de saúde dessa população; Monitoramento e avaliação de indicadores e metas } \\
\text { para a promoção da saúde da mulher, e redução das iniquidades regionais e locais. }\end{array}$ \\
\hline Rede Cegonha & $\begin{array}{l}\text { Importância da portaria que institui a Rede Cegonha como estratégia para implementar rede de cuidados que assegure às } \\
\text { mulheres o planejamento reprodutivo e a atenção humanizada à gravidez, ao parto e ao puerpério, e às crianças o direito } \\
\text { ao nascimento seguro e ao crescimento e ao desenvolvimento saudáveis; Objetivos, diretrizes e componentes da Rede } \\
\text { Cegonha; Rede cegonha no âmbito regional e local; Protocolos assistenciais do pré-natal de risco habitual e de alto risco. }\end{array}$ \\
\hline $\begin{array}{l}\text { Infecções Sexualmente } \\
\text { Transmissíveis (IST) }\end{array}$ & $\begin{array}{l}\text { Importância das IST; Repercussões das IST na transmissão vertical e para a saúde de homens e mulheres; Medidas de } \\
\text { prevenção e controle das IST; Protocolo clínico e as principais diretrizes terapêuticas na atenção integral às pessoas } \\
\text { com IST; Manejo integral das IST assintomáticas (rastreamento e tratamento); Manejo integral das IST sintomáticas } \\
\text { (fluxograma); Panorama das IST nos cenários nacional, regional e local; Papel da rede de saúde e da vigilância } \\
\text { epidemiológica das IST; Informação e comunicação em saúde comunitária relacionada às IST. }\end{array}$ \\
\hline $\begin{array}{l}\text { Direitos Sexuais e } \\
\text { Reprodutivos }\end{array}$ & $\begin{array}{l}\text { Importância dos direitos sexuais e reprodutivos sob a perspectiva de identidade de gênero; Necessidades, riscos e } \\
\text { vulnerabilidade sociais e de saúde da população e como critério de planejamento de ações sobre direitos sexuais } \\
\text { e reprodutivos; Principais estratégias para o planejamento familiar e seus benefícios; Legislação no Brasil sobre } \\
\text { planejamento familiar; Métodos contraceptivos (características, vantagens, desvantagens e contraindicações na } \\
\text { escolha de cada método); Critérios a serem considerados junto ao usuário para definir o melhor método; Exames para } \\
\text { recomendação do método contraceptivo; Principais riscos à saúde associados aos tipos de métodos contraceptivos. }\end{array}$ \\
\hline
\end{tabular}

abordado, considerando artigos, capítulos de livros e manuais institucionais $5,13,17,19$.

Na etapa seguinte, de avaliação da garantia de preparo, que ocorre na sala de aula, as atividades foram desenvolvidas buscando checar e garantir que o estudante estava preparado e pronto para resolver testes individualmente e para contribuir com a sua equipe. Esta fase foi desenvolvida respeitando as quatro etapas constantes da literatura ${ }^{5,8,13,17,19}$

Na primeira fase da etapa 2, fez-se o teste de garantia do preparo individual (Individual Readiness Assurance Test $i R A T)$, com duração de 10 a 15 minutos, no qual os estudantes responderam, sem consulta a qualquer material bibliográfico ou didático, ao teste com dez questões de múltipla escolha (quatro alternativas) sobre os conceitos mais relevantes das leituras indicadas previamente e preenchendo um gabarito individual (Figura 2) 5,8,13,17,19.

Na segunda fase da etapa 2, seguimos para a garantia do preparo em grupo (Group Readiness Assurance Test - gRAT), com duração de 35 a 45 minutos. Os estudantes foram separados nos pequenos grupos previamente definidos para discutir as questões respondidas anteriormente. Cada aluno defendia sua resposta para o grupo, argumentando as razões para sua escolha até que o grupo decidisse qual seria a melhor resposta, utilizando em seguida um instrumento contendo o gabarito que permitia um feedback imediato (Figura 3) 5,8,13,17,19.

\section{IDENTIFICAÇÃO DO GRUPO:}

Instruções: Vocês devem agora discutir as questões entre os membros do grupo, e cada membro deve defender e argumentar as razões para sua escolha até o grupo decidir qual é a melhor resposta.

Após a discussão da questão e decisão da equipe por uma opção, retire a etiqueta correspondente à opção escolhida. Na resposta correta estará impressa a imagem de uma "carinha alegre" e, caso apareça uma "carinha triste", retomem a discussão para escolher outra opção e repitam o procedimento até acertarem.

\section{Como fazer a pontuação da equipe:}

Uma etiqueta retirada: 3 pontos; duas etiquetas retiradas: 2 pontos; três etiquetas retiradas: 1 ponto; quatro etiquetas retiradas: 0 ponto

$90 \mid$\begin{tabular}{l|l} 
REVISTA BRASILEIRA DE EDUCAÇÃO MÉDICA \\
42 (4) : $86-95 ; 2018$
\end{tabular} 
Figura 2

Exemplo de gabarito individual

\section{NOME DO ALUNO:}

\section{IDENTIFICAC̣̃̃O DO GRUPO:}

Instruções: Você está recebendo um questionánio individual onde cada questão vale 4,0 pontos e possui quatro alternativas. A distribuição dos pontos deve ser feita de acordo com a sua decisão e assim você pode optar por colocar os 4,0 pontos em uma só alternativa, ou se inseguro quanto a resposta correta, pode dividir os pontos entre as opções da forma que preferir (Ex: $2+2 ; 3+1 ; 1+2+1 ; 1+1+1+1)$ desde que a soma totalize quatro.

\begin{tabular}{|c|c|c|c|c|c|c|}
\hline $\begin{array}{c}\text { N Questão } \\
\text { Alternativa }\end{array}$ & A & B & C & D & $\begin{array}{l}\text { Pontos } \\
\text { Individual }\end{array}$ & $\begin{array}{l}\text { Pontos } \\
\text { Equipe }\end{array}$ \\
\hline 01 & & & & & & \\
\hline 02 & & & & & & \\
\hline 03 & & & & & & \\
\hline 04 & & & & & & \\
\hline 05 & & & & & & \\
\hline 06 & & & & & & \\
\hline 07 & & & & & & \\
\hline 08 & & & & & & \\
\hline 09 & & & & & & \\
\hline 10 & & & & & & \\
\hline $\begin{array}{c}\text { Total de } \\
\text { pontos }\end{array}$ & & & & & & \\
\hline
\end{tabular}

Fonte: Os autores.

O processo de feedback imediato pode ser feito por meio de cartões de correção instantânea (CCI), do tipo "raspadinhas", por meio de programa eletrônico criado para esse fim ou de alguma outra forma improvisada, desde que preservando os objetivos propostos. Nesse caso, os docentes optaram por um gabarito coberto por etiquetas a serem retiradas de acordo com a resposta acordada em grupo. Nos cartões, a alternativa correta era indicada pelo símbolo de uma "carinha alegre", e as incorretas pelo símbolo de uma "carinha triste" (Figura 3). Assim, ao retirar a etiqueta correspondente à resposta de consenso do grupo, os estudantes poderiam acertar ou não a questão. Quando a resposta escolhida não correspondia à alternativa correta, os estudantes tinham que discutir nova opção, realizando nova tentativa de acerto com a retirada de outra etiqueta, num processo que se repetia até que conseguissem acertar a questão. Nessa etapa, vale destacar que a pontuação, considerando o que preconiza o método, era calculada pelo número de tentativas realizadas pelo grupo até conseguir chegar à alternativa correta $5,8,13,17,19$.

Na terceira fase da etapa 2, com duração de 10 a 20 minutos, era oferecida a oportunidade da apelação, ou seja, nos casos em que o grupo discordava da resposta indicada como
Figura 3

Exemplo de gabarito em grupo, com feedback imediato

\begin{tabular}{|c|c|c|c|c|c|}
\hline $\begin{array}{l}\mathrm{N}^{\circ} \text { questão } \\
\text { alternativa }\end{array}$ & A & B & $\mathrm{C}$ & D & $\begin{array}{l}\text { Pontos } \\
\text { obtidos }\end{array}$ \\
\hline 01 & & & & & \\
\hline 02 & & & & & \\
\hline 03 & & & & & \\
\hline 04 & & & & & \\
\hline 05 & & & & & \\
\hline 06 & & & & & \\
\hline 07 & & & & & \\
\hline 08 & & & & & \\
\hline 09 & & & & & \\
\hline 10 & & & & & \\
\hline $\begin{array}{l}\text { Total de } \\
\text { pontos }\end{array}$ & & & & & \\
\hline
\end{tabular}

correta pelos docentes, os estudantes tinham a possibilidade de questionar formalmente essa resposta, desde que de forma escrita, contendo indicação de referências e evidências científicas que sustentem o texto de argumentação da equipe. Assim, durante as sessões, as equipes foram informadas sobre essa possibilidade, embora tenham se limitado a pequenas contestações verbais após as sessões, sendo prestados esclarecimentos pelos docentes ${ }^{5,8,13,17,19}$.

Na quarta e última fase da etapa 2, cuja duração depende da necessidade, os professores/facilitadores realizaram feedback comentando os gabaritos. Ressalte-se que o feedback deve ser constante. Assim, sempre que o facilitador sentir que deve intervir na discussão do grupo sem causar interferência no raciocínio ou crescimento dos estudantes, deverá fazê-lo. Podem ser realizadas breves exposições dialogadas sobre os principais conceitos que os conteúdos propostos no tema de discussão do TBL trouxeram, tentando esclarecer as principais dúvidas e oferecer uma devolutiva aos estudantes $5,8,13,17,19$. 
Na etapa 3 do $T B L$, de aplicação de conceitos, os estudantes, ainda reunidos em equipes, recebiam questões apresentadas sob a forma de cenários/problemas relevantes e presentes na prática profissional diária, no formato verdadeiro ou falso, para responder em grupo e depois realizar a argumentação entre as equipes. Os professores/facilitadores deveriam exigir dos estudantes a aplicação dos conceitos aprendidos anteriormente, o que poderia ser feito com um teste de múltipla escolha, questão do tipo verdadeiro ou falso e casos clínicos, entre outros.

Nessa fase, obedeceu-se aos quatro princípios básicos da aplicação de conceitos. Aqui, os estudantes foram desafiados a fazer interpretação, inferência, análises ou síntese, sendo cada princípio explicitado da seguinte forma ${ }^{13}$ :

- Problema significativo (Significant Problem): O problema a ser solucionado deveria ser real, contendo situações com que o aluno poderia se deparar no dia a dia da prática profissional, levando a interpretação, inferência e análise da situação-problema;

- Escolha específica (Specific Choice): As alternativas deveriam ser claras e específicas, no formato de múltipla escolha. Por exemplo: Qual destes é o melhor exemplo de X? O que é a evidência mais importante da Y? Qual destas evidências mais se aplica a $Z$ ? Nunca se deve pedir às equipes que produzam respostas escritas em longos documentos;

- Mesmo problema (Same Problem): Todas as equipes deveriam receber o mesmo problema e ao mesmo tempo. Assim, trabalhando com o mesmo problema, cada equipe se preocupava com as conclusões e fundamentos das outras equipes;

- Relatos simultâneos (Simultaneously Report): A simultaneidade dos relatos tornava possível explorar os efeitos instrucionais ideais entre as equipes. Assim, as respostas eram mostradas simultaneamente, para que os grupos não utilizassem a argumentação de outras equipes em suas respostas. Nesta etapa, cada equipe tinha que se comprometer com sua resposta e defendê-la na argumentação com as outras equipes em caso de divergência.

Os momentos presenciais em sala de aula tinham duração média de três horários de 50 minutos cada. Ao término, como forma de incentivo aos estudantes, todas as equipes recebiam um brinde simbólico, sendo que aquela(s) com melhor desempenho tinha(m) direito a um segundo melhor brinde.

A avaliação do desempenho foi realizada por meio da quantidade de acertos do grupo, o que era contabilizado e divulgado no final de cada aula. No final do módulo, realizou-se um extrato da média individual e do grupo, obtida em todas as aulas para compor a avaliação formativa do módulo.
A etapa 4 - autoavaliação e avaliação interpares dos estudantes para observar o cumprimento dos objetivos da discussão do TBL -, embora considerada de grande relevância, em nosso caso não foi realizada, sendo essa uma fragilidade em nossa experiência.

Ressaltamos que, segundo Ferreira ${ }^{16}$, é muito importante tanto para o professor quanto para o estudante que haja a avaliação dos pares, pois ajuda o professor a avaliar quais alunos cumpriram ou não os objetivos da sua disciplina e quais se prepararam adequadamente no momento pré-classe. Afinal, é neste momento que cada aluno faz a autoavaliação e avalia cada um dos demais integrantes do próprio grupo.

\section{AVALIAÇÃO DA INSERÇÃO DA ESTRATÉGIA TBL}

Os estudantes se mostraram extremamente motivados com o método durante a aplicação, sendo possível perceber que eles compreendiam ter responsabilidades perante seus pares não somente durante o preparo pré-classe, mas também nos momentos presenciais, quando tinham que explicar e fundamentar suas respostas. Isto está de acordo com Pamelee et al. ${ }^{20}$, que afirmam que o processo do $T B L$ produz responsabilização de todos tanto pelo trabalho individual quanto por sua contribuição para a equipe.

Ao acompanhar as discussões em grupo, ficava claro aos docentes quais estudantes tinham realmente realizado seu preparo pré-classe individual, pois na argumentação facilmente percebia-se o desempenho de cada aluno. Para isso, o quantitativo de docentes envolvidos na atividade foi um facilitador, uma vez que cada grupo era acompanhado por um docente, que procurava incentivar a interação e a participação de todos na argumentação em equipe. Ressalte-se que é tarefa do instrutor acessar os espaços da sala para perceber como as equipes estão processando a tarefa e usar o que ouviu nas discussões da aula em sequência ${ }^{20}$.

Observou-se que o engajamento do aluno se deu em dois contextos, concordando com autores que ressaltam esse envolvimento durante a interação com o material ofertado mediante questionamentos, hipóteses e conexão com novos conhecimentos e dentro das equipes pelo trabalho em conjunto pela oportunidade de melhoria da aprendizagem durante a troca de informações da equipe para chegar a decisões de consenso ${ }^{8,20,21}$.

Foi possível perceber que os estudantes, no processo de continuidade das aulas, após as etapas 1 e 2 do método TBL, mostravam-se mais estimulados para adquirir e construir seus conhecimentos. Ao exercitarem suas habilidades de comunicação, argumentação e convencimento, melhoraram sua interação com as opiniões dos colegas em um processo de colaboração entre pares e crescimento pessoal. 
Essa melhoria é de grande relevância se considerarmos que o mundo do trabalho contemporâneo exige uma formação de profissionais com perfil crítico-reflexivo e capazes de trabalhar em equipes ${ }^{22}$. Além disso, para qualquer futuro médico, aprender a trabalhar em colaboração com os outros é uma das competências mais importantes para seu domínio ${ }^{20}$.

Outro aspecto percebido pelos docentes durante a continuidade da utilização do método foi o surgimento de uma sutil competição entre os estudantes, mas que não foi vista como desfavorável ao processo de aprendizagem; ao contrário, observou-se que os estudantes se sentiam mais motivados a participar, tornando o ambiente de aprendizado mais interessante e reduzindo o desinteresse dos mesmos. Para Barbiero et al., ${ }^{23}$, o TBL traz benefícios para os alunos e torna-os mais ativos nas aulas, além de instigá-los a buscar novos conhecimentos referentes ao assunto, tornando-os mais independentes no estudo.

A produção coletiva nesse ambiente de aprendizagem colaborativa também foi valorizada, pois era nítida a busca de um desempenho melhor para a equipe. Ao final, observou-se que o desempenho da equipe tendia a superar os desempenhos individuais, inclusive frequentemente superando o desempenho isolado do melhor membro da equipe no resultado obtido na avaliação do teste de garantia do preparo individual, o que também é apontado na literatura ${ }^{8,20,21}$. Além disso, o grupo apresentou melhoras progressivas com o decorrer das aulas, talvez por ter superado a ideia de grupo em que o conjunto de pessoas trabalha por um mesmo objetivo, mas onde é comum haver competição entre os membros, para um trabalho em equipe, onde os membros trabalham em prol de um único objetivo e cada membro colabora para essa conquista ${ }^{16}$.

As divergências em relação aos gabaritos surgiram durante as atividades do módulo de concepção, formação do ser humano e saúde reprodutiva, que deram espaços para apelações que, ao serem pensadas e elaboradas, contribuíram para reflexão e habilidade de comunicação e arguição dos discentes. O professor deve inclusive fazer uma avaliação que incite as equipes a realizar apelação, como utilizar duas alternativas corretas, sendo uma "mais correta" que a outra, pois assim $\mathrm{o}$ aluno pesquisa na literatura e tem chance de $\operatorname{argumentar}^{16}$.

$\mathrm{Na}$ avaliação, percebeu-se um ponto de fragilidade do processo de uso do $T B L$, pois os estudantes eram avaliados pelo seu desempenho individual e também pelo resultado do trabalho em grupo, mas não foram submetidos à avaliação entre os pares, o que poderia incrementar a responsabilização e colaboração entre eles. Afinal, a avaliação por pares, segundo Krug et al. ${ }^{24}$, permite que os alunos comparem sua autoavaliação com a avaliação de outros alunos, pois o feedback dos colegas tem o potencial de ajudá-los a desenvolver impressões mais precisas de si mesmos e de suas habilidades, da cooperação em grupo e da comunicação.

Outras fragilidades observadas durante a programação foram relacionadas a questões ligadas à coesão interna nos grupos. Isto porque, por um lado, a formação de vínculos afetivos entre componentes e a expertise diferenciada de alguns desses membros, e, de outro lado, a presença de desconfortos quando alguns estudantes individualmente não se preparavam adequadamente, pouco contribuindo para o desempenho de sua equipe, resultaram em ressentimentos de alguns estudantes que se prepararam de forma mais adequada e se sentiram sobrecarregados. Isto poderia ter sido mais bem trabalhado e abordado com a avaliação interpares.

Contudo, apesar dessas dificuldades, o TBL representou uma estratégia pedagógica que valoriza a responsabilidade individual dos estudantes perante as suas equipes de trabalho na construção dos próprios conhecimentos. Motivou o estudo e aplicação dos conhecimentos adquiridos na solução de questões relevantes no contexto da prática em outros ambientes de aprendizagem do curso.

Sabe-se que que o volume de conhecimento biomédico aumenta implacavelmente. Em paralelo, as horas de contato entre professores e alunos não podem ser expandidas. Para resolver esse dilema, os educadores confiam cada vez mais em estratégias que organizam o conhecimento essencial em formatos acessíveis para o aprendizado independente e fora da sala ${ }^{21}$. O TBL, nesse sentido, torna o tempo presencial mais proveitosamente utilizado, pois envolve alunos e professores em um diálogo com foco na garantia da aquisição de conhecimentos prévios e de sua melhoria.

Além disso, a prática da medicina está se tornando cada vez mais interprofissional e voltada ao trabalho em equipe. Nesse sentido, o TBL mostrou-se como relevante ferramenta, pois em cada uma de suas fases as equipes heterogêneas são estimuladas a pensar em conjunto sobre inúmeros e variados problemas relacionados à prática médica.

Neste relato, uma das limitações presentes é a de não existir uma avaliação formal do impacto dessa aplicação para retenção de conteúdos a médio e longo prazo. Entretanto, em estudo com revisão sistemática de 117 artigos que avaliam o uso de TBL em profissões da saúde, 79\% dos estudos compararam os resultados de aprendizagem do TBL com outro método de ensino, mais comumente a aula expositiva, e a maioria descobriu que o TBL produziu melhores resultados acadêmicos ${ }^{8}$.

\section{CONSIDERAÇÕES FINAIS}

Nesse contexto, observa-se que o uso do TBL no módulo de concepção, formação do ser humano e saúde reprodutiva foi bastante 
útil no processo de aprendizagem e na avaliação da formação. Permitiu sistematizar e organizar os conceitos e conhecimentos apresentados aos estudantes no processo de aprendizagem e na avaliação. Acredita-se que o TBL permitiu ofertar de forma mais articulada os conhecimentos necessários para responder às demandas e necessidades de saúde mais frequentes da população e auxiliar na transformação da realidade das condições de vida e saúde locais. Porém, essa prática ainda não está sistemicamente inserida em todos os ambientes de aprendizagem do curso.

A relação verticalizada, onde o professor transmite as informações e os estudantes as absorvem, deve dá lugar à troca de visões, em que o docente assume o papel de condutor do ensino como facilitador, tirando dúvidas, aprofundando o tema e estimulando o debate, de forma a proporcionar ao estudante um aprendizado mais amplo e completo.

Os benefícios observados nas competências adquiridas pelos estudantes foram: troca colaborativa do conhecimento, descoberta de ampla gama de estratégias de resolução de problemas, habilidades de comunicação e argumentação, melhoria da interdependência positiva e aplicação imediata dos conceitos apresentados durante o momento presencial em sala de aula.

No entanto, observa-se a necessidade de estudos complementares que investiguem como o TBL vem sendo aplicado nos cursos da saúde e que verifiquem o impacto a médio e longo prazo de sua aplicação para a retenção dos conteúdos no ensino de saúde.

\section{REFERÊNCIAS}

1. Silva, LS, Cotta RMM, Costa GD, Oliveira Campos AA, Cotta RM, Silva LS, et al. Formação de profissionais críticos-reflexivos: o potencial das metodologias ativas de ensino aprendizagem e avaliação na aprendizagem significativa. Revista del Congrés Internacional de Docència Universitària i Innovació (CIDUI) 2014; (2).

2. Mitre SM, Siqueira-Batista R, Girardi-de-Mendonça JM, Morais-Pinto NMD, Meirelles CDAB, Pinto-Porto C, et al. Metodologias ativas de ensino-aprendizagem na formação profissional em saúde: debates atuais. Ciência \& saúde coletiva2008; 13 (suppl.2): 2133-2144.

3. Oliveira Costa RR, Medeiros SM, Martins JCA, Menezes RMP, Araújo MS. O uso da simulação no contexto da educação e formação em saúde e enfermagem: uma reflexão acadêmica. Espaço para a Saúde-Revista de Saúde Pública do Paraná 2015; 16 (1): 59-65.

4. Pandini CMC, Roesler J. Métodos, Estratégias e Procedimentos Didáticos. Palhoça: Unisul, 2007.
5. Bollela VR, Senger MH, Tourinho, FSV, Amaral E. Aprendizagem baseada em equipes: da teoria à prática. Medicina (Ribeirão Preto. Online) 2014; 47(3): 293-300.

6. Souza CDS, Iglesias AG, Pazin-Filho A. Estratégias inovadoras para métodos de ensino tradicionais: aspectos gerais. Medicina (Ribeiräo Preto) 2014; 284-292.

7. Dias RF. Team-based learning: fazendo os alunos pensarem "fora da caixa", os elementos essenciais para sua implantação. Revista Brasileira de Educação e Saúde 2015; 5(1): 75-81.

8. Tyler Reimschisel, Anna L. Herring, Jennifer Huang \& Tara J. Minor. A systematic review of the published literature on team-based learning in health professions education. Medical Teacher, 2017; 39:12, 1227-1237.

9. Júnior GAP, Santos MA, Bernardi FA, Ferreira WD, Senju AA, Paula Jesus TH, et al. Desenvolvimento de Plataforma Digital para Ensino de Graduação (Caso do ensino de atendimento ao paciente traumatizado). Revista de Graduação USP 2017; 2(1):13-23.

10. Morán J. Mudando a educação com metodologias ativas. PROEX/UEPG, 2015.

11. Sharma N, Lau CS, Doherty I, Harbutt D. How we flipped the medical classroom. Medical teacher 2015; 37(4):327-330.

12. Moffett J. Twelve tips for "flipping" the classroom. Medical teacher 2015; 37(4): 331-336.

13. Krug RDR, Vieira MSM, Maciel MVDA, Erdmann TR, Vieira FCDF, Koch MC, et al. The "Bê-Á-Bá" of Team-Based Learning. Revista Brasileira de Educação Médica 2016; 40(4): 602-610.

14. Fundação Getúlio Vargas. Sala de aula invertida. EI! Ensino Inovativo 2015; (Volume Especial), 14-17.

15. Michaelsen LK, Knight AB, Fink LD. Team-based learning: A transformative use of small groups. Greenwood publishing group, 2002.

16. Ferreira ASSBS. Aprendizagem Baseada em Equipes: da teoria à prática. Botucatu: NEAD, 2017.

17. Farias PAMD, Martin ALDAR, Cristo CS. (2015). Aprendizagem ativa na educação em saúde: percurso histórico e aplicações. Rev Bras Educ Méd 2015; 39(1): 143-150.

18. Torres PL, Irala EA. Aprendizagem colaborativa: teoria e prática. Curitiba: SENAR-PR, 2014.

19. Oliveira TE, Araujo IS, Veit EA. Aprendizagem Baseada em Equipes (Team-Based Learning): um método ativo para o Ensino de Física. Caderno Brasileiro de Ensino de Física 2016; 33(3): 962-986.

20. Parmelee D, Michaelsen LK, Cook S, Hudes PD. Team-based learning: a practical guide. AMEE guide no. 65. Med Teach 2012;34(5):e275-e7. 
21. Koles, Paul G. MD; Stolfi, Adrienne; Borges, Nicole J; Nelson, Stuart; Parmelee, Dean. The Impact of Team-Based Learning on Medical Students' Academic Performance. Academic Medicine, 2010, Vol. 85, No. 11, 1739-1745.

22. Bandeira, Denise Maria Almeida; Silva, Maria Alexsandra; Vilela, Rosana Quintella B. Aprendizagem Baseada em Equipe. Revist. Port.: Saúde e Sociedade. 2017;2(1):371-379.

23. Barbiero, Adriana Jordão Costa; Honorato, Andreia Assante; Vieira, Cristiane Ferrari; da Silva, Glauco Teixeira Gomes; Ferreira, Igor Monteze; Barreiros, Lívia Lopes; Lopes e Silva, Luiz Felipe; Lopes e Silva, Mara Lúcia Farias; Segheto, Wellington; Fontes, Lívia Beatriz Almeida. Percepção dos acadêmicos de medicina sobre a metodologia de aprendizagem baseada em equipes na disciplina de farmacologia. Revista Científica Fagoc - Saúde. 2017, 2(2): 43-49.

24. Krug, Rodrigo de Rosso, Vieira, Maria Salete Medeiros, Maciel, Marcus Vinicius de Andrade e, Erdmann, Thomas Rolf, Vieira, Fábio Cavalcanti de Faria, Koch, Milene Caroline, \& Grosseman, Suely. (2016). O “Bê-Á-Bá" da Aprendizagem Baseada em Equipe. Revista Brasileira de Educação Médica, 40(4), 602-610.

\section{CONTRIBUIÇAO DOS AUTORES}

Bruno Luciano Carneiro Alves de Oliveira, Sara Fiterman Lima e Livia dos Santos Rodrigues, participaram igualmente de todas as fases de elaboração do manuscrito.

Gerson Alves Pereira Júnior realizou a orientação e revisão da versão final manuscrito.

\section{CONFLITO DE INTERESSES}

Não existe

\section{ENDEREÇO PARA CORRESPONDÊNCIA}

Universidade Federal do Maranhão, Estrada Pinheiro, Pacas, km 10, s/n, Enseada, CEP 65200-000, Pinheiro, Maranhão, Brasil. 